Original Research Paper

\title{
A Novel Four-Directional Thresholding Approach for Lung Computed-Tomography Images by Using Similarity-Based Segmentation Technique
}

\author{
Saleheh Heidari, Muhamad Taufik Abdullah and Lili Nurliana Abdullah \\ Department of Multimedia, Faculty of Computer Science and Information Technology, University Putra Malaysia, 43400, \\ UPM Serdang, Malaysia
}

Article history

Received: 23-01-2014

Revised: 28-04-2014

Accepted: 08-08-2014

Corresponding Author: Muhamad Taufik Abdullah Department of Multimedia, Faculty of Computer Science and Information Technology,

University Putra Malaysia, 43400, UPM Serdang, Malaysia

Email: saleheh.heidari@gmail.com

\section{Introduction}

Lung is the most complex and bilateral organ in human body and is enclosed by mediastinum, diaphragm and musculoskeletal chest wall (Ganti and Gerber, 2013). It comprises arteries, blood capillaries, bronchi and pulmonary veins and fulfills the task of intrathoracic air-exchange. Therefore, the healthiness of lung and keeping that away from any respiratory diseases is vitally important for human life. Diffuse Parenchymal Lung Disease (DPLD) and generally Interestitial Lung Diseases (ILD) are known as prevalent lung disease in which the lung

\begin{abstract}
In automated pulmonary nodules extraction and lung disease diagnosis by image processing techniques, image segmentation is utilized as a primary and the most essential step of lung tumor analysis. But due to extensive similarity between pulmonary vessels, bronchus and arteries in lung region and the low contrast of the ComputedTomography (CT) image the accuracy of lung tumor diagnosis is highly dependent on the precision of segmentation. Therefore, precise lung CT image segmentation has become a challenging preprocessing task for every lung disease pathological application.In this study, a novel FourDirectional Thresholding (FDT) technique is introduced. This propounded technique segments the pulmonary parenchyma in Computed-Tomography (CT) images using the Similarity-Based Segmentation (SBS). The proposed technique aims to augment the precision of the $\mathrm{CT}$ image thresholding by implementing an advanced thresholding approach from four different directions in which the determination of pixels' value as being either on foreground or background is highly dependent on its adjacent pixel's intensity value and the final decision is made based on all four directions' thresholding results. In this study the importance of neighbor pixels in precision of thresholding with FDT technique is demonstrated and the effectiveness of FDT method has been evaluated on different CT inages. Eventually the result of segmentation using FDT method is compared by other precursors techniques, which corroborates the high exactitude of proposed technique.
\end{abstract}

Keywords: CT Image Segmentation, Lung Parenchyma Extraction, Similarity-Based Segmentation, Thresholding parenchyma and its interstitial part is highly affected and its dissemination will result in respiratory failure (Guo et al., 2002; Helen et al., 2011). Recently, the prevalence and mortality due to lung disease and specially lung cancer has been gradually increased. The studies done in United States shows that the lifetime risk of invasive lung cancer for women is approximately about 5.5 and $5.9 \%$ for men. It is argued that over $80 \%$ of lung cancer could be cured if the disease is diagnosed in early stage (Julien, 2013).

Lung cancer is a type of pulmonary disease in which anomalous cells propagating and forming a tumor. Pulmonary nodules are acting as the early phase of lung 
diseases (Yue et al., 2011). Therefore, the growth characteristics of the nodules must be evaluated in order to define the malignancy or benignity of the tumor. Determining the type of tumor could be applicable by performing the morphological analysis on extracted nodules. Though, lung nodules extraction and malignant tumor detection is highly challenging and associates with some difficulties, but its extraction would not be possible without using image processing techniques.

Image processing techniques can be adopted as a useful tool for identification and diagnosis of lung tumors as the primary sign of the lung diseases. Computed-Tomography (CT) has been exploited as a standard instrument for pulmonary nodules detection. The vast majority of minor lung diseases are visible in the CT images, despite the fact that due to low contrast of the CT image the extraction of lung nodules would be a challenging task. Determining the Region Of Interest (ROI) and extracting the pulmonary nodules in $\mathrm{CT}$ images requires image segmentation.

Considering that the unreliable thresholding would thwart the accurate morphological analysis of pulmonary nodules, many researchers have been urged to propose a segmentation technique, which results a precise thresholding. As it is perused, previous studies merely attempted to obtain a specific Threshold value (T) by particular algorithms and then thresholding the image based on that value. Therefore, the process of thresholding after reaching the " $\mathrm{T}$ " value remained the same in previous studies.

In this study a novel thresholding technique is proposed in which the implementation of " $T$ " value on image demonstrates that the way in which the thresholding is done has more impact on thresholding precision that " $\mathrm{T}$ " value optimization.

The proposed FDT technique aims to segment the lung region by performing a novel four-directional thresholding approach on CT image in order to prepare the image for nodule extraction and any further analysis. The process of performing the thresholding based on proposed approach is defined in following sections and the result is compared with other thresholding methods. The framework of this study is organized as follow.

Prior studies and their achievements are looked over in section 2. In section 3, the proposed FDT approach is profoundly expounded. The results and performance evaluation of new FDT technique are described in section 4. Ultimately the study is concluded in section 5 and the future work is conferred.

\section{Related Work}

Segmentation technique is simply construed into two different categories namely contextual or non-contextual. Thresholding is specified as a non-contextual technique in which the color or gray-scale image is transformed into binary region map (Efford, 2000). Generally, segmentation is the most pragmatic preprocessing technique that could be applied on manifold filed where the texture of background or foreground should be analyzed (Golchin et al., 2013). As the failure and success rate of every image processing applications is extremely dependent on reliability and scrupulousness of the preprocessing techniques and especially thresholding, therefore, many researchers attempted to introduce thresholding optimization techniques using algorithms such as P-tile (Doyle, 1962), Gray-Level Histogram thresholding "Otsu" (Otsu, 1979), Maximum Entropy with adaptive Genetic algorithm (Li and Nie, 2009), Shanbhag algorithm (Shanbhag, 1994), Yen technique (Yen et al., 1995), Entropic thresholding method based on Ant Colony Genetic algorithm (Shen et al., 2009), integration of clustering algorithm and marker controlled watershed segmentation algorithm (Christ et al., 2010) and many other algorithms.

Despite the techniques that are used for various purposes, the maximum class-between variance known as "Otsu" technique is the most famed and prominent approach that many researchers used it as a cardinal technique.

Otsu technique takes the gray-level of pixels and their local average for determining the optimal value for thresholding (Otsu, 1979). In Spite of the complexity of the computation for gaining the optimum $\mathrm{T}$ value by Otsu, but the result is not impeccable for some CT images with low contrast. Therefore, several studies attempted to optimize the $\mathrm{T}$ value by enhancing the Otsu technique.

Helen et al. (2011) posited the pulmonary parenchyma extraction from multi-sliced CT image based on improving the Otsu method. Their study investigated the optimization of Otsu technique based on Particle Swarm Optimization (PSO) approach. In order to gain the appropriate $\mathrm{T}$ value by PSO technique, the algorithm firstly calculates the statistics of the image based on obtained gray level, then the iteration count is set and particles are initialized using defined function. When the fitness value is evaluated for each particle, their velocity and position are modified until the iteration count gains the maximum value. Then the image is thresholded using the reached $\mathrm{T}$ value. However, in their proposed technique the result is almost identical to Otsu, but the 
computational cost lessened to approximately less than 1 sec per slice.

Subsequent to PSO method, Zhang and Zhou (2012) introduced an advanced PSO approach by combining both Otsu and PSO algorithm and enhanced the execution time and precision of results by improving the particle's fitness value as the inertia weight of PSO to adjust the velocity of the particles. By taking the advantage of both Otsu and PSO approach, Nickfarjam et al. (2012) came up with a supervised Bi-Level Thresholding method using Otsu based on PSO technique. In Bi-Level thresholding approach, the capability of minimizing the withinclass variance of Otsu method is used to obtain a better result. Therefore, to balance and uniform the combination of Otsu and PSO (Nickfarjam et al., 2012) attempted to perform preprocessing to define a canonical image before they apply the PSO method to reach the optimum threshold value. As it is demonstrated by their experimental results, their proposed methodenhanced the thresholding precision with less computational time and complexity. Beyond the several attempts on enhancing the PSO technique, there are many efforts to boost the quality and thoroughness of thresholding by improving Otsu technique.

In segmentation algorithm based on Otsu method represented by (Huang et al., 2012) the selection of optimum thresholding value is based on minimum variance ratio which results better computational speed as well as satisfactory precision in compare to Otsu. On the other hand ( $\mathrm{Lu}$ and $\mathrm{Hu}, 2012$ ) proposed a combination method of Otsu and Ant Colony Optimization (ACO) algorithm in which the behavior of ants to finding a closest path from their colony to food resources has been mimicked in order to obtain a sufficient thresholding outcome. As it was demonstrated is asserted in their study the computation time is reduced significantly in compare to Otsu method. Since the result of Three-Dimensional (3D) Otsu was not adequate in many cases, Wang et al. (2012) postulated another segmentation algorithm based on improvement of Three-Dimensional Otsu thresholding. The propounded technique aims to enhance the 3D Otsu algorithm by providing a fast iteration method based on gray level gradient and improvement of regional division and it came out with a great computation time reduction by approximately $80 \%$ in compare to standard 3D Otsu segmentation.

Nevertheless, great efforts have been done to achieve the optimum threshold value; some algorithms would not give a pertinent result on medical CT images. Therefore, Ebrahimdoost et al. (2010) presented a medical image segmentation approach to deal with improper algorithm. Ebrahimdoost et al. (2010) used active contour and a level set model to design a thresholding approach for pulmonary embolism segmentation. Since level-set segmentation is not pragmatic with low contrast image, they attempted to resolve the problem by taking the advantages of hybrid speed function that is formulated based on image gradient and intensity. The assessment of their technique endorses the efficiency of their technique. In addition, another automatic lung parenchyma segmentation proposed by (Liming et al., 2011). This new automatic algorithm repairs the boundary of lung region by using mathematical morphology method. In this method the CT image is thresholded based on Global thresholding approach that properly segments the image and prepares that for further morphological analysis. In order to segment sub-solid pulmonary nodules, Nie et al. (2012) presented a new method based on Fuzzy C-means Clustering defied as Improved Weighted Kernel Fuzzy C-Mean (IWKFCM). The developed algorithm is practical to identify and cluster the potential nodule pixels located in ROI. Performance of the technique indicates the smaller False-Negative (FN) and FalsePositive (FP) rate. In investigation done by (Sivakumar and Chandrasekar, 2012), taking the advantage of Fuzzy C-Means (FCM) and Weighted Fuzzy C-Means (WFCM) algorithm results an accurate and precise segmentation in medical CT image.

In summary, every researcher attempted to optimize the $\mathrm{T}$ value rather than applying the different thresholding technique. Thus, the novel FDT technique is proposed in order to indicate the significance of implementation of thresholding against simple thresholding with optimized $\mathrm{T}$ value. Considering all studies, the Otsu method (Otsu, 1979) and improved Otsu by PSO algorithm (Helen et al., 2011) could have better result on CT image Thresholding. Therefore, the proposed FDT technique will be compared with these two algorithms in following sections.

\section{Materials and Methods}

\section{Image Segmentation}

Recently, manifold approaches on image segmentation have been proposed as an important step of preprocessing in image understanding. Thresholding (segmentation) an image is the most fundamental and indispensable problem in image processing, which also considered as a significant function in computer vision. Segregating the image's object from its background could be accomplished by either 
Similarity-Based (SB) or Discontinuity-Based (DB) segmentation. Discontinuity-based technique merely identifies isolated pixels, edges and lines by applying specific mask on sub-images. However, Similaritybased approach attempts to group those pixels that are similar in intensity. Thresholding is one of the most renowned similarity-based segmentation techniques.

The importance of image thresholding is to reduce the amount of data in an image in order to obtain the meaningful information for further analysis. Furthermore, it aims to simplify the shifting representation of an image into more expressive manner to make it more comprehensible for further analysis. Thus, it is the most dominant part of nodule extraction in CT image as it helps labeling the CT image's pixels and assigns them into same label type with common visual characteristics (Sharma and Jindal, 2011).

\section{Proposed Four-Directional Thresholding (FDT) Approach}

The FDT algorithm performs thresholding on CT image in four different directions, in which any direction's result can affect the final step of thresholding process. The FDT technique involves 5 steps in which the image is thresholded from four different directions and then their results are matched base on the algorithm decision.

\section{Image Characteristics}

Considering a CT image with $\mathrm{M} * \mathrm{~N}$ size (Fig. 1), in which $M$ represents the number of pixels in $y$-axis (number of rows) and $\mathrm{N}$ indicates the number of pixels in X-axis (number of columns). Each pixel's location is represented by $\mathrm{P}(\mathrm{r}, \mathrm{c})$. It should be noted that the number of pixels in rows and columns are always M-1 and N-1 Respectively.

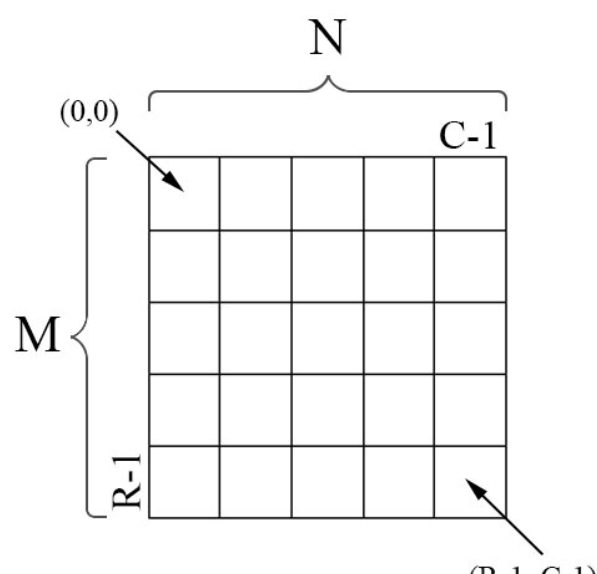

(R-1, C-1)

Fig. 1. Basic image structure

\section{FDT Method}

Step 1: The initial step of this technique is to find the ' $T$ ' value (Thresholding Value) by calculating the arithmetic mean (Average) of all pixels intensity values Equation 1:

$T=\frac{1}{M N} \sum_{r=0}^{M-1} \sum_{c=0}^{N-1} P(r, c)$

Prior to proceed with the main part of the technique, be aware of the pixels' boundary. In all steps the formula would be performed in the specific ranges of pixels in which Pixel Intensity Value (PIV) would only be calculated in the range of $r>M-1$ and $c<N-1$ and for any pixels out of this range (normally the pixels located at the edge of the image) the PIV is calculated as below Equation 2:

$P I V=\frac{1}{2}[P(r, c)+P(r, c)]$

Step 2: In this step the algorithm performs its task from left to right (First Direction), in which the average of each pixel and its adjacent pixel to the right will be compared with $\mathrm{T}$ value Equation 3:

$P(r, c)=\left\{\begin{array}{l}1, \text { if } P I V=\frac{1}{2}[P(r, c)+P(r, c+1)] \geq T \\ 0, \text { if else }\end{array}\right.$

Step 3: The 'Second Direction' would be contrary to the previous one, in which the algorithm performs its task in opposite direction from right to left. In this step $P(r, c-1)$ should be added by its left adjacent pixel Equation 4:

$P(r, c)=\left\{\begin{array}{l}1, \text { if } P I V=\frac{1}{2}[P(r, c-1)+P(r, c)] \geq T \\ 0, \text { if else }\end{array}\right.$

Step 4: In 'Third Direction' the algorithm performs its task from top to bottom and the pixel should be added by its bottom-adjacent Equation 5:

$P(r, c)=\left\{\begin{array}{l}1, \text { if } P I V=\frac{1}{2}[P(r, c)+P(r-1, c)] \geq T \\ 0, \text { if else }\end{array}\right.$

Step 5: The 'Fourth Direction' accomplishes in opposite direction, from bottom to top of the image. In this way the $P(r-1, c)$ must be added by its topneighbor pixel Equation 6: 


$$
P(r, c)=\left\{\begin{array}{l}
1, \text { if } P I V=\frac{1}{2}[P(r-1, c)+P(r, c)] \geq T \\
0, \text { if else }
\end{array}\right.
$$

Step 6: Last step so-called decision-making, ponders on every individual directions to make the final decision for thresholding. Here, if the value of $P(r, c)$ in more than 2 steps remains identical then the pixel would remain identical.

\section{Experimentation}

In fulfillment of proposed FDT technique, all the six stated steps have been accomplished on the portion of $\mathrm{CT}$ image to meticulously clarify the process involved in performing the FDT algorithm Fig. 2.
Table 1 shows the pixels' intensity value of $9 * 9$ portion of the $\mathrm{CT}$ image presented in Fig. 2. Each pixel represents a particular intensity value of the 0 255 range of gray-scale level of the CT image, which significantly affect on thresholding result by FDT technique.

Figure 3 elucidates the results of each steps that FDT algorithm comprises. As the first step of FDT implementation is to define the $\mathrm{T}$ value, the thresholding value is determined by averaging the pixels value given in Table 1. Which results in 69.67 as the $\mathrm{T}$ value. Therefore the considered portion is segmented by the given mean value $(\mathrm{T})$. The final thresholding result (Fig. $3 \mathrm{E}$ ) is determined based on the result in $\mathrm{A}, \mathrm{B}, \mathrm{C}$ and $\mathrm{D}$. Where black pixels iterated in more than 2 steps, they remain identical for final result.

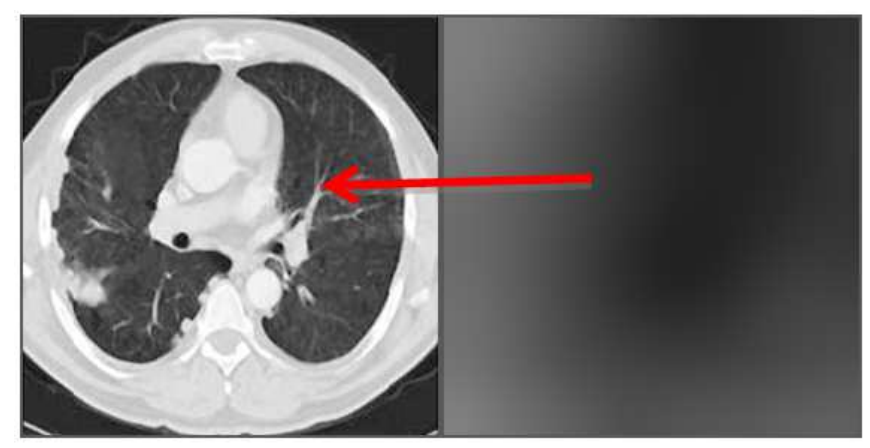

(a)

(b)

Fig. 2. (A) CT image, (B) Small portion of CT image

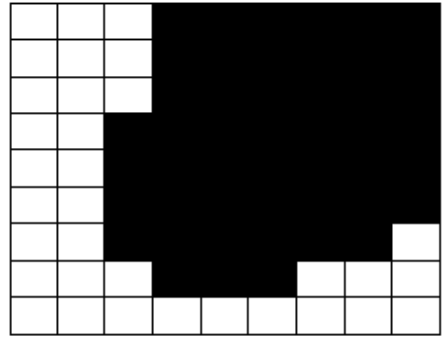

(A)

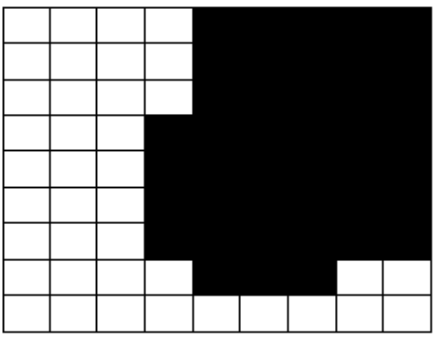

(B)

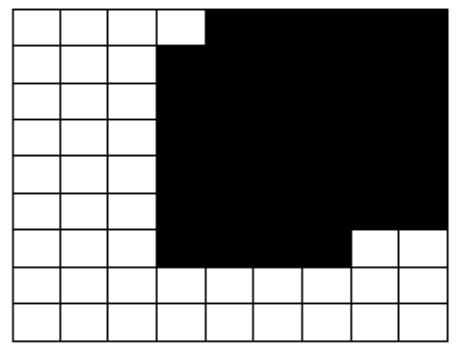

(C)

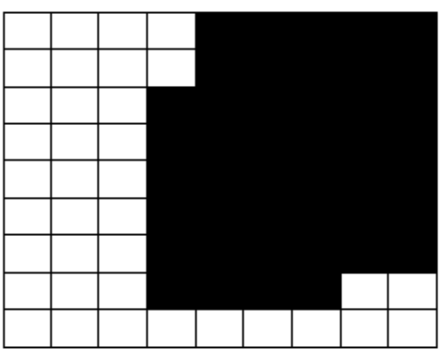

(D)

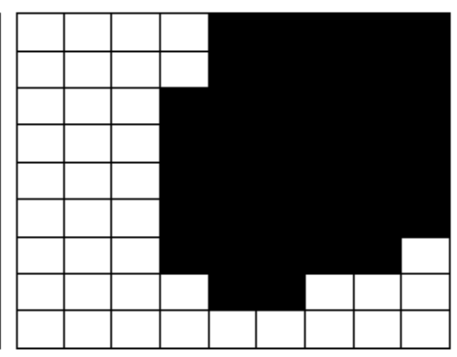

(E)

Fig. 3. (A) First direction from left to right, (B) second direction from right to left, (C) third direction from top to bottom, (D) forth direction from bottom to top. (E) final result (matching step) 


\begin{tabular}{lrrrrrrrr}
\multicolumn{1}{c}{ Table 1. Pixels' intensity value of the digital CT image in Fig. 2B } \\
\hline 125 & 117 & 101 & 79 & 53 & 36 & 34 & 40 & 47 \\
123 & 111 & 91 & 69 & 49 & 36 & 35 & 39 & 49 \\
117 & 102 & 82 & 62 & 46 & 37 & 36 & 39 & 50 \\
109 & 96 & 78 & 60 & 45 & 36 & 36 & 41 & 50 \\
105 & 93 & 75 & 56 & 40 & 33 & 38 & 47 & 52 \\
106 & 93 & 73 & 53 & 38 & 35 & 45 & 58 & 59 \\
110 & 96 & 76 & 60 & 49 & 48 & 57 & 68 & 70 \\
113 & 99 & 82 & 71 & 65 & 64 & 68 & 73 & 78 \\
125 & 110 & 97 & 82 & 78 & 83 & 84 & 90 & 93
\end{tabular}

\section{Result}

As discussed earlier, lung nodule extraction in CT images is highly convoluted and dependent on the precision of the thresholding. Normally, Lung CT images are lacking of brightness and contrast and it would cause the loss of enormous pixels, which tending the lung nodule extraction process toward inaccurate detection due to inexact thresholding. For that reason retaining even small area of interest signifies the thoroughness of segmentation.

Generally, for determining the lung diseases and discerning the malignancy or benignity of lung tumor, the characteristics of extracted nodules must be analyzed by morphological analysis techniques. In this case, every single pixel might have a significant effect on determining the types of tumor. Therefore, a precise thresholding technique must be applied on CT image. In this study a FDT technique was introduced in which the use of four directions helps to maintain important nodules in lung lobes and also removes some unwanted data, which are considered as noise.

Figure 4 shows the results of all four directions of FDT algorithm on small portion of CT image in order to magnify the better representation of the FDT performance. Considering the images in $\mathrm{A}, \mathrm{B}, \mathrm{C}$ and $\mathrm{D}$ that represented the performance of every direction respectively, some small noises could be seen that the final step of FDT technique eliminated them as unwanted pixels.

In order to examine the reliability and efficiency of proposed thresholding Technique (FDT), the result of the FDT is compared with both Otsu and advanced PSO method in Fig. 5.

\section{Performance Evaluation}

Proposed FDT technique reveals that how scrupulousness of performing the thresholding on CT image could have an influence on precision and reliability of the thresholding result.

\section{Region Non-Uniformity}

Despite the visual comparison represent by experimental results in Fig. 5, it is extremely crucial to assess the algorithm performance by evaluation criterion (Soltaninejad et al., 2012).

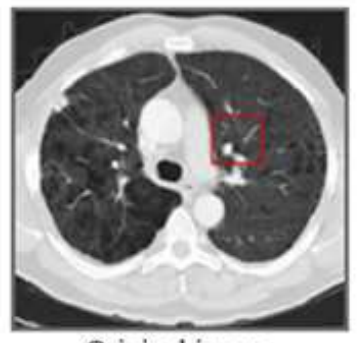

Original image

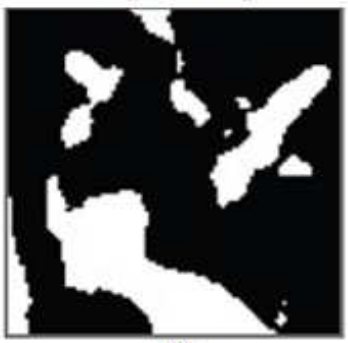

(A)

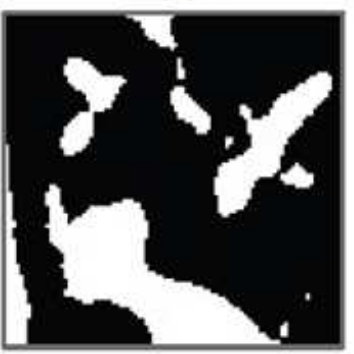

(C)

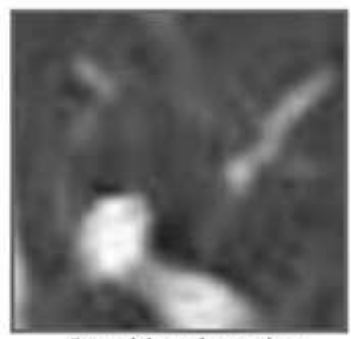

Considered portion

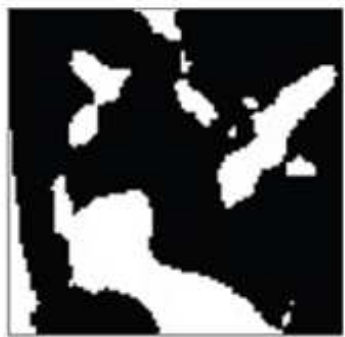

(B)

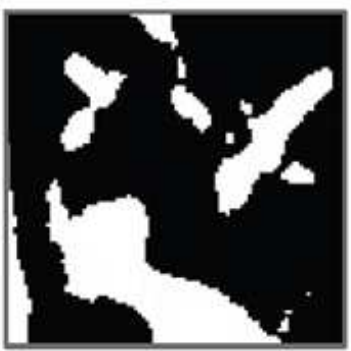

(D)

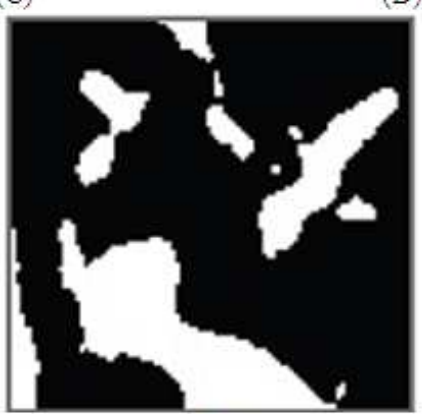

(E)

Fig. 4. (A) First direction from left to right, (B) second direction from right to left, (C) third direction from top to bottom, (D) forth Direction from bottom to top. (E) final result (matching step)

Therefore, Region Non-Uniformity of the threshold image will be evaluated by following Equation 7:

$R N U=\left(\frac{F_{T}}{F_{T}+B_{T}}\right) \times\left(\frac{\sigma_{t}^{2}}{\sigma^{2}}\right)$

where, $F_{T}$ and $B_{T}$ are foreground and background pixels' area, respectively; and variance of foreground and whole image is represented by $\sigma_{t}^{2}$ and $\sigma^{2}$. 


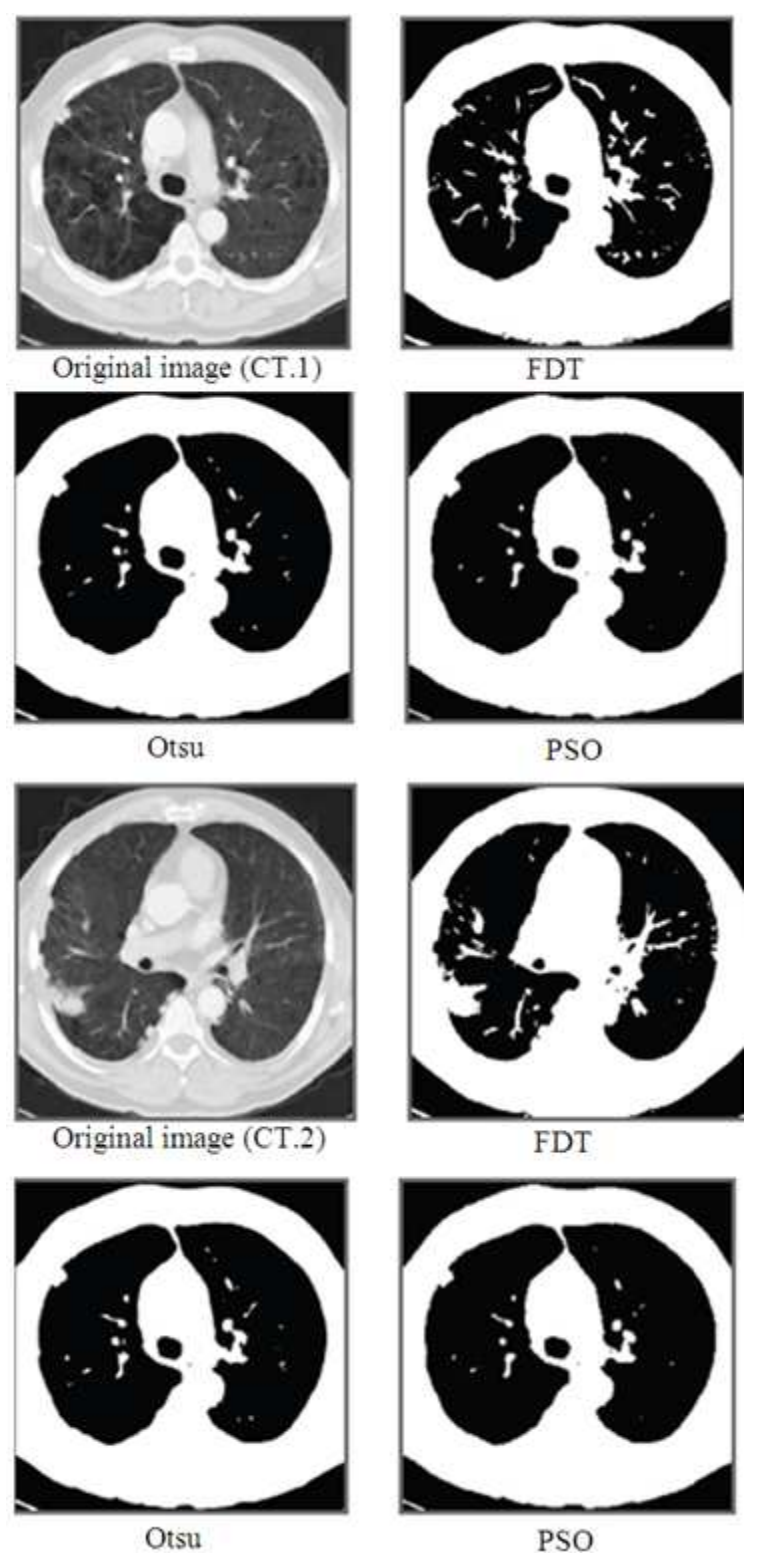

Fig. 5. Comparison of FDT technique with Otsu method and Otsu Improved by PSO in different CT images 1 and 2

Table 2. Evaluation result using Region Non-Uniformity

\begin{tabular}{lll}
\hline & Region Non-Uniformity & \\
& CT image 1 & CT image 2 \\
\hline Otsu & 0.1406 & 0.1387 \\
PSO & 0.1400 & 0.1386 \\
FDT & 0.1372 & 0.1306 \\
\hline
\end{tabular}

Table 3. Evaluation result using Correlation

\begin{tabular}{lll}
\hline & Correlation & \\
& CT image 1 & CT image 2 \\
\hline Otsu & 0.9237 & 0.9272 \\
PSO & 0.9061 & 0.9221 \\
\hline
\end{tabular}

It is noticeable that the value of Region NonUniformity evaluation denotes the quality of segmentation. If the value is closed to " 0 " it represents the well-performed and precise thresholding and if the value is near to " 1 " it indicates the unreliable thresholding.

Table 2 shows the Region Non-Uniformity of FDT, Otsu and PSO methods for images shown in Fig. 5.

\section{Correlation}

Assessment of the performance by correlation technique denotes that how two images are closely related to each other. In this evaluation, the image threshold by FDT is compared with the results of other two techniques in Fig. 5, by following Equation 8:

$$
C=\frac{\sum_{m} \sum_{n}\left(A_{m n}-\bar{A}\right)\left(B_{m n}-\bar{B}\right)}{\sqrt{\left[\sum_{m} \sum_{n}\left(A_{m n}-\bar{A}\right)^{2}\right]\left[\sum_{m} \sum_{n}\left(B_{m n}-\bar{B}\right)^{2}\right]}}
$$

where, $\bar{A}$ and $\bar{B}$ represents mean value in corresponding images.

Table 3 represents how the result of FDT technique is related to Otsu and $\mathrm{PSO}$.

\section{Discussion}

Conspicuously, thresholding plays a cardinal role as an initial preprocessing step in every pathological application specially lung tumor diagnosis. Owing to the fact that lung nodules' morphological characteristic has significant influence on tumor diagnosis, the proposed FDT thresholding technique augmented the precision of segmentation process by implementing a novel fourdirectional algorithm.

In preceding algorithms like Otsu and PSO, the researchers strive to solely optimize the $\mathrm{T}$ value, while FDT technique attempts to perform the thresholding process in which neighbor pixels' intensity value has been considered in every steps of algorithm. The comparison results of FDT with Otsu and PSO method manifest the improvement of the thresholding and its exactitude performance in compare to previous techniques.

The given evaluations' results corroborate that FDT has reduced the regional non-uniformity by approximately 2.42 and $2 \%$ compared to OTSU and PSO respectively, in CT Image 1 and about 5.84 and 5.77\% reduction of non-uniform region compared to OTSU and PSO in CT image 2.

\section{Conclusion}

In this study a novel Four-Directional Thresholding (FDT) technique was introduced for lung CT image thresholding by taking advantage of similarity-based segmentation approach. The FDT technique optimizes the 
precision of thresholding result by implementing the algorithm from four different directions and the enhanced result asserted the performance of the proposed technique in comparison with Otsu and PSO thresholding techniques. The proposed FDT technique focuses on implementing the $\mathrm{T}$ value on $\mathrm{CT}$ image to threshold image by different approach rather than merely optimizing the $\mathrm{T}$ value. The result of FDT performance indicates its exactitude and extensive precision on lung CT images. In this study the FDT approach has been proposed for Lung ComputedTomography images, but it can be implemented on various medical images and the thresholding value could be optimized further in future works as required.

\section{Funding Information}

The authors have no support or funding to report.

\section{Author's Contributions}

All authors equally contributed in this work.

\section{Ethics}

This article is original and contains unpublished material. The corresponding author confirms that all of the other authors have read and approved the manuscript and no ethical issues involved.

\section{References}

Christ, F., A. Voet, A. Marchand, S. Nicolet and B.A. Desimmie et al., 2010. Rational design of smallmolecule inhibitors of the ledgf/p75-integrase interaction and HIV replication. Nat. Chem. Biol., 6: 442-448. PMID: 20473303

Doyle, W., 1962. Operation useful for similarity-invariant pattern recognition. J. Assoc. Comput. Machinery, 9: 259-267. DOI: $10.1145 / 321119.321123$

Ebrahimdoost, Y., J. Dehmeshki, T.S. Ellis, M. Firoozbakht and A. Youannic et al., 2010. Medical image segmentation using active contours and a level set model: Application to Pulmonary Embolism (PE) segmentation. Proceedings of the Fourth International Conference on Digital Society, Feb. 10-16, IEEE Xplore Press, St. Maarten, pp: 269-273. DOI: 10.1109/ICDS.2010.64

Efford, N., 2000. Digital Image Processing: A Practical Introduction Using Java. 1st Edn., Addison-Wesley, Harlow, ISBN-10: 0201596237, pp: 340.

Ganti, A.K. and D.E. Gerber, 2013. Lung Cancer. 1st Edn., Oxford University Press, USA, ISBN10: 0199935939, pp: 39.

Golchin, M., F. Khalid, L.N. Abdullah and S.H. Davarpanah, 2013. Shadow detection using color and edge information. J. Comput. Sci., 9: 1575-1588. DOI: $10.3844 /$ jcssp.2013.1575.1588
Guo, J., J.M. Reinhardt, H. Kitaoka, L. Zhang and M. Sonka et al., 2002. Integrated system for CT-based assessment of parenchymal lung disease. Proceedings of the IEEE International Symposium on Biomedical Imaging, Jul. 7-10, IEEE Xplore Press, pp: 871-874. DOI: 10.1109/ISBI.2002.1029398

Helen, R., N. Kamaraj, K. Selvi and V. Raja Raman, 2011. Segmentation of pulmonary parenchyma in CT lung images based on 2D Otsu optimized by PSO. Proceedings of the International Conference on Emerging Trends in Electrical and Computer Technology, Mar. 23-24, IEEE Xplore Press, Tamil Nadu, pp: 536- 541. DOI: 10.1109/ICETECT.2011.5760176

Huang, M., W. Yu and D. Zhu, 2012. An improved image segmentation algorithm based on the Otsu method. Proceedings of the 13th ACIS International Conference on Software Engineering, Artificial Intelligence, Networking and Parallel and Distributed Computing, Aug. 8-10, IEEE Xplore Press, Kyoto, pp: 135-139. DOI: 10.1109/SNPD.2012.26

Julien, P.J., 2013. CT Lung Screening. Cedars-Sinai.

Li, S. and S. Nie, 2009. Image segmentation method of heavy forgings based on genetic algorithm. Processing of the 2nd International Congress on Image and Signal, Oct. 17-19, IEEE Xplore Press, Tianjin, pp: 1-4. DOI: 10.1109/CISP.2009.5303977

Liming, D., Z. Caiming and G. Shanshan, 2011. A new algorithm of automatic lung parenchyma segmentation based on CT images. Proceeding of the International Conference on Consumer Electronics, Communications and Networks, Apr. 16-18, IEEE Xplore Press, XianNing, pp: 976-979. DOI: 10.1109/CECNET.2011.5768258

Lu, J. and R. Hu, 2012. A new image segmentation method based on Otsu method and ant colony algorithm. Proceedings of the International Conference on Computer Science and Information Processing, Aug. 24-26, IEEE Xplore Press, Xi'an, Shaanxi, pp: 767769. DOI: $10.1109 /$ CSIP.2012.6308966

Nickfarjam, A.M., S. Soltaninejad and F. Tajeripour, 2012. Supervised bi-level thresholding based on particle swarm optimization. Proceedings of the 16th CSI International Symposium on Artificial Intelligence and Signal Processing, May 2-3, IEEE Xplore Press, Shiraz, Fars, pp: 370- 373. DOI: 10.1109/AISP.2012.6313775

Nie, S., L. Li, Y. Wang, C. He and F. Ji et al., 2012. A segmentation method for sub-solid pulmonary nodules based on fuzzy c-means clustering. Proceedings of the 5th International Conference on Biomedical Engineering and Informatics, Oct. 1618, IEEE Xplore Press, Chongqing, pp: 169-172. DOI: 10.1109/BMEI.2012.6513127 
Otsu, N., 1979. A threshold selection method from graylevel histograms. IEEE Trans. Syst. Man Cybernet., 9: 62-66. DOI: 10.1109/TSMC.1979.4310076

Shanbhag, A.G., 1994. Utilization of information measure as a means of image thresholding, CVGIP: Graphical Models Image Process., 56: 414-419. DOI: 10.1006/cgip.1994.1037

Sharma, D. and G. Jindal, 2011. Identifying lung cancer using image processing techniques. Proceeding of the International Conference on Computational Techniques and Artificial Intelligence, (TAI' 11), pp: 115-120.

Shen, X., Y. Zhang and F. Li, 2009. An improved twodimensional entropic thresholding method based on ant colony genetic algorithm. Proceedings of the WRI Global Congress on Intelligent Systems, May 19-21, IEEE Xplore Press, Xiamen, pp: 163-167. DOI: 10.1109/GCIS.2009.96

Sivakumar, S. and C. Chandrasekar, 2012. Lungs image segmentation through weighted FCM. Proceedings of the International Conference on Recent Advances in Computing and Software Systems, Apr. 25-27, IEEE Xplore Press, Chennai, pp: 109113. DOI: 10.1109/RACSS.2012.6212707

Soltaninejad, M.R., R.A. Zoroofi and G. Shirani, 2012. Automatic crown surface reconstruction using tooth statistical model for dental prosthesis planning. Proceedings of the 19th Iranian Conference of Biomedical Engineering, Dec. 20-21, IEEE Xplore Press, Tehran, pp: 218-222.

DOI: 10.1109/ICBME.2012.6519684
Wang, Q., H. Zhao, W. Wu and N. Yuan, 2012. Algorithm for segmentation based on an improved three-dimensional Otsu's thresholding. Proceedings of the 2nd International Conference on Computer Science and Network Technology, Dec. 29-31, IEEE Xplore Press, Changchun, pp: 1737-1740. DOI: $10.1109 /$ ICCSNT.2012.6526256

Yen, J.C., F.J. Chang and S. Chang, 1995. A new criterion for automatic multilevel thresholding. IEEE Trans. Image Process., 4: 370-378. DOI: 10.1109/83.366472

Yue, L., L. Jie and M. Lingjun, 2011. Suspected pulmonary nodule detection algorithm based on morphology and gray entropy. Proceedings of the IEEE International Conference on Computer Science and Automation Engineering, Jun. 10-12, IEEE Xplore Press, Shanghai, pp: 103-108. DOI: 10.1109/CSAE.2011.5952812

Zhang, Z. and N. Zhou, 2012. A novel image segmentation method combined Otsu and improved PSO. Proceedings of the IEEE 5th International Conference on Advanced Computational Intelligence, Oct. 18-20, IEEE Xplore Press, Nanjing, pp: 583-586. DOI: 10.1109/ICACI.2012.6463232 